\title{
LAS DEMOSTRACIONES DINÁMICAS DEL TEOREMA DE PITÁGORAS
}

\author{
María Consuelo Barrantes Masot, Víctor Zamora Rodríguez \\ y Manuel Barrantes López
}

\begin{abstract}
RESUMEN. Presentamos un estudio sobre demostraciones algebraicas y geométricas del Teorema de Pitágoras, para la Enseñanza Secundaria, usando software libre de geometría dinámica. La categorización de las demostraciones ofrece al docente un muestrario útil como herramienta para la selección de los recursos didácticos necesarios para la enseñanza y aprendizaje de dicho teorema. El conjunto de construcciones dinámicas representadas con GeoGebra, como resultado de una revisión actualizada y una selección posterior podría favorecer la tarea docente del profesor.
\end{abstract}

\begin{abstract}
AвSTRACт. We present a study on algebraic and geometric proofs of the Pythagorean Theorem, for Secondary Education, using free software of dynamic geometry. The categorization of the demonstrations offers the teacher a useful sample as a tool for the selection of the didactic resources necessary for the teaching and learning of this theorem. The set of dynamic constructions represented with GeoGebra, as a result of an updated revision and a subsequent selection, could favour the teacher's teaching task.
\end{abstract}

\section{§1. Introducción}

Durante muchos años se le ha atribuido a Pitágoras (585-500 a.C.), filósofo y matemático griego, el enunciado y demostración del teorema geométrico que lleva su nombre, el cual expresa la relación entre los cuadrados construidos sobre los lados de un triángulo rectángulo. Existen evidencias de que en otras culturas también se conocía el teorema aunque no se conoce la existencia de su demostración. Se asegura (Bergua, 1958) que durante sus viajes a Egipto y al oriente antiguo, el sabio griego conoció el enunciado de la regla y se dedicó a demostrarla.

Palabras clave: Teorema de Pitágoras, Software de geometría dinámica, Enseñanza de la geometría, Demostraciones geométricas.

Keywords: Theorem of Pythagoras, Dynamic geometry software, Teaching Geometry, Geometrical demonstrations. 
El enunciado que dieron los griegos al Teorema de Pitágoras es el siguiente: el área del cuadrado construido sobre la hipotenusa, de un triángulo rectángulo es igual a la suma de las áreas de los cuadrados construidos sobre los catetos. El enunciado moderno usa términos algebraicos: en un triángulo rectángulo, el cuadrado de la hipotenusa es igual a la suma de los cuadrados de los catetos. Euclides, según su interpretación al teorema, dice que el área del cuadrado cuyo lado es la hipotenusa es la suma de las áreas de los cuadrados cuyos lados son los catetos.

Esta celebre proposición, conocida como el Teorema de Pitágoras, la proposición pitagórica o la proposición 47 del primer libro de los Elementos de Euclides, ha jugado un papel fundamental en el desarrollo de la Matemática. La relación pitagórica es la ecuación de la circunferencia que aparece constantemente en el estudio de la Geometría Analítica y de la Trigonometría, y el teorema del coseno es un caso particular de dicho teorema. También es la raíz histórica del análisis indeterminado de Diofanto y Fermat, y la fuente de casi todas las relaciones métricas en Geometría.

Es notorio el valor práctico del teorema en todas las Ciencias, concretamente en el campo de la Física se encuentra en la rama de Mecánica Clásica, Astrofísica, Física del Estado Sólido, Electromagnetismo, entre otros.

Nuestro trabajo se orienta principalmente a resaltar el interés didáctico de dicha proposición en la enseñanza y aprendizaje de la matemática, y en particular de la Geometría. Así pues, el objetivo general de este aporte para la enseñanza ha sido el de presentar de manera selectiva, demostraciones dinámicas, entendidas como aquellas en las que el alumno manipula los elementos bien manualmente o mediante el ordenador. Estas demostraciones pretendemos contribuyan a la enseñanza de dicho teorema como resultado de una revisión actualizada. Además de la manipulación, queremos mostrar a la comunidad docente la necesidad de utilizar metodologías de enseñanza en las que se haga uso de software de geometría dinámica.

Para cumplir este objetivo se ha realizado una aproximación mediante una revisión bibliográfica, así como una exploración de las construcciones dinámicas del teorema que ofrecen varias webs educativas incluidas en la webgrafía como por ejemplo:

http: / / elcuadradodelahipotenusa.blogspot.com.es,

http: //www.cut-the-knot.org/pythagoras/index.shtml

A partir de este material, se ha realizado una selección y clasificación de las demostraciones dinámicas encontradas, a las que hemos añadido construcciones propias que complementan la revisión general y añaden un componente didáctico a fin de propiciar adaptaciones para el aula. 
§2. Acerca de la construcción de las reglas de cálculo para sumar

La historia de la Matemática, como lugar de encuentro entre las ciencias y las humanidades, es un instrumento magistral para enriquecer culturalmente la enseñanza de la Matemática en el aula (González, 2004; Alsina, Fortuny, y Burgués, 1988; Alsina, 1997, 2008). Ahora bien, cuando las actividades se reducen a la inclusión de la biografía de un personaje determinado o de algún hecho aislado relacionado con la materia a tratar, entonces el atractivo y la utilidad de los recursos históricos para la enseñanza quedan muy limitados.

Una aproximación más directa al aprovechamiento matemático de estos recursos en la enseñanza se puede conseguir proponiendo al alumnado, diferentes demostraciones clásicas de la proposición o problemas relacionados con ella, transportando a éstos a la época en las que fueron realizadas, apelando a las tecnologías disponibles, mediante las demostraciones que veremos en nuestro estudio.

Para ello, los profesores en sus clases, además de comentar la biografía de Pitágoras (Bergua, 1958; Schuré, 1995; Caniff, 1997; Strathern, 1999) pueden examinar qué métodos utilizaban para las diferentes demostraciones utilizando los recursos que se describen en este trabajo como son los puzles y GeoGebra.

Por ejemplo, (González, 2001, 2008) presenta el pensamiento de Pitágoras de una forma integrada. Se destacan los logros matemáticos del filósofo griego en el contexto de los restantes aspectos del Pitagorismo. Se presentan los antecedentes del Teorema de Pitágoras en civilizaciones como la Prehelénica, la Babilónica, en la India, Egipto y China, así como las implicaciones y desarrollo de su pensamiento, no sólo en la Historia de las Matemáticas, sino también en la Historia general. En este último sentido, el plural recorrido de las enseñanzas pitagóricas a través del tiempo resulta uno de los aspectos más interesantes de estos trabajos de González.

En (Barrantes, 1998) se realiza el estudio de dicho teorema desde el punto de vista histórico, tratando de estudiar o conocer la vida de Pitágoras y los pitagóricos. También se trabajan diferentes demostraciones de la proposición a lo largo de la historia que son curiosas o pertenecen a algún matemático conocido, así como las repercusiones y aplicaciones de dicha proposición para el avance de la Matemática.

Por ejemplo, (Nelsen, 1993), con el objetivo principal de proporcionar pistas en cada demostración para estimular el pensamiento matemático, presenta una recopilación de demostraciones matemáticas a través de imágenes o diagramas. Sin embargo, una de las recopilaciones más importante la hace (Loomis, 1968), en una reedición del mismo texto de 1940. Encontramos 370 demostraciones diferentes numeradas y clasificadas como: algebraicas, geométricas, vectoriales y otras que utilizan la dinámica. Las dos principales vías de demostración del teorema, es decir: la analítica o algebraica y la visual o geométrica consideramos son las más adecuadas para la utilizarlas en la Enseñanza Secundaria (12-16 años). 
Por otra parte, dentro de los matemáticos, físicos e ingenieros ilustres de la historia que abordaron el Teorema de Pitágoras no falta el genio del Renacimiento, Leonardo da Vinci que presenta una demostración basada en equivalencias de polígonos y que también es nombrada en (Loomis, 1968) con el número 46. También es el caso de la demostración realizada por James A. Garfield (clasificada con el número 23) que tiene la particularidad de ser muy ingeniosa y puede servir al profesor para relacionar la propiedad pitagórica con el cuadrado de la suma de un binomio, también estudiado en estos cursos.

Para ilustrar que el teorema era conocido por geómetras anteriores a los pitagóricos, sería estimulante considerar dos demostraciones más, idóneas para la Enseñanza Secundaria: la atribuida al matemático indio Bhaskara y (ver ejemplo 3 de este artículo) y una demostración china que nos muestra (Loomis, 1968) con los números 353 y 36.

En (Barrantes, 1998) se presentan también tres demostraciones basadas en puzles de seis piezas, de cinco piezas y tres piezas. La principal curiosidad del puzle de tres piezas radica en que es el de menor número de piezas que nos permite mostrar la propiedad pitagórica. En dicho estudio se considera el teorema como un problema abierto, accesible a los alumnos y a la vez motivante, mediante la utilización de recursos y materiales apropiados como son los puzles pitagóricos con los que se pueden realizar diferentes demostraciones del teorema, e incluso el alumno puede construir su propia demostración en consonancia con las demostraciones geométricas de (Loomis, 1968).

Llegados a este punto, se puede ampliar el estudio de la proposición pitagórica, pues no es válida únicamente para la suma de las áreas de los cuadrados construidos sobre los catetos que nos da el área del cuadrado de la hipotenusa, sino también para cualquier tipo de polígonos construidos sobre los lados del triángulo rectángulo, siempre que los tres polígonos, así construidos, sean semejantes entre sí. Concretamente, (Barrantes, 1998) muestra la propiedad ampliada a triángulos semejantes, rombos, trapecios y otras figuras irregulares. En la misma línea (Vasquez, 2012) presenta demostraciones para distintos casos como: rectángulos semejantes, triángulos equiláteros y semejantes, que se generalizan a polígonos irregulares semejantes, como figuras siempre triangulables, y en el límite, a semicírculos entendidos como polígonos de infinitos lados.

Esto nos indica que la generalización de la propiedad no es sólo para polígonos, sino para figuras cualesquiera que verifiquen la condición de semejanza. Así pues, los alumnos pueden comprobar que se verifica para semicírculos, cuartos de círculos, segmentos o sectores circulares. 


\section{§3. Uso del software de GeoGebra para la enseñanza de geometría}

Los diferentes currículum en las legislaciones educativa vigentes hacen mención a la competencia digital, al tratar de forma adecuada la información y, en su caso, servir de apoyo a la resolución de problemas y comprobación de la solución. Consideramos la geometría dinámica como un ambiente computacional de construcción geométrica, basado en la geometría euclidiana. Este recurso se fundamenta en la tecnología y las herramientas que nos proporciona, ya que a través de ellas podemos movilizar las figuras geométricas para que adquieran dinamismo (Cabrera y Campistrous, 2007).

Con esta nueva concepción de la enseñanza de la geometría, se evitan las figuras rígidas que se corresponden con una única forma de representación. Sin embargo, mediante esta metodología, los alumnos, pelando a un gran número de representaciones, se pueden hacer una idea más precisa de las figuras y llegar a comprender mejor las propiedades geométricas.

Así pues, consideramos que los programas informáticos de geometría dinámica, son un recurso importante que se ponen a disposición de los estudiantes para realizar las demostraciones dinámicas. (Groman, 1996) afirma que la geometría dinámica hace que el alumno aprenda las demostraciones de forma que construye por sí mismo los significados matemáticos en un contexto donde el profesor es un participante más del proceso.

Las demostraciones dinámicas hacen que el alumno explore las figuras geométricas para producir conjeturas. (Fiallo, 2010) señala que los estudiantes, descubren los conceptos y propiedades con gran independencia del profesor. Esto los motiva a querer demostrar la validez, y el programa les proporciona herramientas necesarias para hacerlo. (Mariotti, 2000), que trabaja con Cabri, indica que se produce una correspondencia importante entre los comandos del menú y las justificaciones que dan los alumnos de manera que se incrementa la necesidad de recurrir a la demostración como un recurso de validación. Para (Marrades y Gutiérrez, 2000), el programa de geometría dinámica permite una exploración empírica de las representaciones de las figuras geométricas, hecho que influye en una evolución positiva hacia la producción de justificaciones cada vez más próximas a demostraciones deductivas. La explicación de las figuras construidas juega implícitamente con la idea de inferencia y prepara al alumnado para comprender cómo opera una demostración (Jones, 2000).

Otro aspecto importante a resaltar son las interacciones que se producen entre estudiantes y profesor. Distintos autores como (Bell, 1976; Lampert, 1990; Gutiérrez y Jaime, 2012) o (Alibert y Thomas, 1991) sugieren desarrollar las actividades de forma que los estudiantes pueden aceptar o rechazar argumentos independientemente de lo que afirme el profesor. Las estrategias didácticas en las que el alumno 
reflexiona sobre lo que constituye una demostración aceptable en matemática conducen a que el debate sea una forma excelente para discutir y comprender la validez de las premisas, y querer justificarlas mediante la producción de una demostración (Hoyles, 1997; Jones, 2000). La demostración como recurso de comunicación de ideas debe llevarse a cabo a través de actividades que propician la formulación de argumentos para convencer a los demás (De Villiers, 1993; Duval, 1998).

En esta propuesta, los autores utilizan GeoGebra como software de Geometría dinámica. GeoGebra aumenta las posibilidades didácticas pues además de utilizarse como apoyo a las explicaciones, da al alumnado posibilidades para visualizar figuras, modificarlas y construirlas, así como para observar, buscar la solución, describir, conjeturar, comprobar e investigar. En definitiva, actividades para que sean ellos quienes hagan y aprendan a partir de su experiencia más que de los conceptos teóricos que pueda impartirle el profesor (Sada, 2010).

Así pues, el uso de un paquete de geometría dinámica como GeoGebra, permite generar experiencias de aprendizaje en las que los alumnos se pregunten el por qué, y el qué sucedería si... de ciertos hechos geométricos, aportando una oportunidad para desarrollar una base para llegar a una completa apreciación de la naturaleza y el propósito de las demostraciones dinámicas.

Una vez hecho un recorrido bibliográfico del tema central del estudio, en los siguientes apartados, pasaremos a describir el desarrollo para el aporte en la enseñanza.

\section{$\S 4$. Objetivos y metodología}

El objetivo principal de nuestro trabajo es presentar selectivamente construcciones dinámicas que contribuyan a la enseñanza del Teorema de Pitágoras en Secundaria haciendo uso de software de geometría dinámica, como resultado de una revisión actualizada.

Es decir, pretendemos realizar una aproximación sobre la enseñanza del Teorema de Pitágoras en la Enseñanza Secundaria y sobre la enseñanza del mismo a través de software de geometría dinámica, concretamente utilizando GeoGebra.

El trabajo se realizó en dos fases, una primera fase de primer contacto con las demostraciones del Teorema de Pitágoras en general, y una segunda enfocada en su selección, clasificación y posterior diseño con el programa GeoGebra. Durante la primera fase se revisaron las demostraciones desechando aquellas que tenían semejanzas con otras, o aquellas que consideramos no adecuadas al nivel educativo al cual fue orientando la investigación, debido a la propia complejidad de éstas. 
Se hizo un estudio bibliográfico en textos como (Barrantes y Balletbo, 2012), revistas y otros documentos, tanto de las bibliotecas de la Universidad de Extremadura como en páginas webs educativas que se encuentran en internet.

La segunda fase fue útil para realizar la selección definitiva de las demostraciones y para ir perfilando su clasificación. La formación de las categorías de análisis (nivel educativo y tipo de demostración: geométrica y algebraica) fue un proceso que se sustentó en la revisión bibliográfica realizada, así como en los objetivos de la investigación. Se han tenido en cuenta, también, aquellas demostraciones tanto algebraicas como geométricas de matemáticos o personas relevantes a lo largo de la historia, y cuyas demostraciones han causado un impacto en el campo de la Geometría.

Para cada una de las demostraciones se presenta una ficha en la que se incluye: cuatro imágenes (momentos); el procedimiento generalizado utilizado para su construcción; una explicación justificativa de la demostración y por último, dicha demostración. Para observar todos los pasos de los que constan las construcciones el alumno puede entrar en la página de GeoGebra creada por los autores de este trabajo (https://www. geogebra.org/m/j6wRRyxB).

Por último, aclarar que las conclusiones e implicaciones del trabajo se sustentan tanto en la bibliografía revisada como en los resultados obtenidos y, en general, en todos los procesos llevados a cabo.

\section{§5. Datos y resultados}

Partiendo de la revisión bibliográfica e internet seleccionamos un total de 97 construcciones dinámicas sobre las demostraciones geométricas y algebraicas del Teorema de Pitágoras. Teniendo en cuenta los niveles del estudio hemos seleccionado 28 demostraciones del Teorema de Pitágoras realizadas con GeoGebra; 10 son demostraciones algebraicas y 18 geométricas. Estas demostraciones, se han clasificado de acuerdo al nivel educativo para el que están orientadas, de forma que seis corresponden a primer curso, doce demostraciones son para segundo y diez para cuarto curso de la Enseñanza Secundaria (12-16 años) como se muestra en la Tabla 1.

Atendiendo al desarrollo madurativo de los alumnos podemos observar que las demostraciones dirigidas a primer curso, son básicamente puzles, de manera que las piezas en que se dividen los cuadrados construidos sobre los catetos deben rellenar todo el área del cuadrado de la hipotenusa. Esta es una forma motivante para trabajar la relación pitagórica, ya que ayuda al alumno a desarrollar un comportamiento más inteligente que de tipo reflejo automático (Barrantes, 1990).

Las demostraciones geométricas dirigidas a segundo curso, son demostraciones realizadas por matemáticos célebres o personalidades de otros campos, que en algún momento de su trabajo han realizado una demostración de dicho teorema. 


\begin{tabular}{|l|l|l|}
\hline \multicolumn{2}{|c|}{ Geométricas } & Algebraicas \\
\hline $\mathbf{1}^{\mathbf{0}}$ ESO & $\mathbf{2}^{\mathbf{0}}$ ESO & $\mathbf{4}^{\mathbf{0}}$ ESO \\
\hline 1. Puzzle 1: & 7. Euclides (L. 33) & 19. Bashkara (L. 36) \\
Hipotenusa/cateto menor $=3$ & 8. Liu Hui (L. 28) & 20. Chou Pei Suan (L. 253) \\
2. Puzzle 2: & 9. Platón (L. 98) & 21. Pappus (L. 42) \\
$30^{\circ} \leq A \leq 60^{\circ}$ y $30^{\circ} \leq B \leq 60^{\circ}$ & 10. Dobriner (L. 18) & 22. Vieta (L. 63) \\
3. Puzzle 3: & 11. Perigal (L. 205) & 23. Garfield (L. 231) \\
Cateto mayor/cateto menor =2 & 12. Ozanam (L. 16) & 24. L. da Vinci (L. 46) \\
4. Puzzle 4: & 13. J. Adams (L. 27) & 25. H. Boad (L. 90) \\
Triángulo rectángulo isósceles & 14. Hoffmann (L. 240) & 26. Loomis 108 \\
5. Puzzle 5: Lados 3, 4 y 5 & 15. Loomis 2 & 27. Thâbit Ibn Qurra (a) \\
6. Caso Particular (L. 4) & 16. Loomis 26 & 28. Thâbit Ibn Qurra (b) \\
& 17. Poo-Sung-Park & \\
\hline & 18. A. G. Samosvat & \\
\hline
\end{tabular}

Tabla 1. Demostraciones seleccionadas del Teorema de Pitágoras (entre paréntesis aparece el número de la demostración de Loomis).

Para la muestra, en este artículo, hemos seleccionados la demostración clásica y muy conocida de Euclides.

Por último, las demostraciones algebraicas de cuarto curso, son también demostraciones realizadas por celebridades de todos los tiempos entre las que hemos elegido la del matemático Bashkara.

Si el lector está interesado, el resto de las demostraciones clasificadas pueden ser consultadas de manera electrónica en el Libro de GeoGebra "Pruebas del Teorema de Pitágoras", en la página web: https://www.geogebra.org/m/j6wRRyxB. También, en la Tabla 1 están reflejadas todas las demostraciones construidas en el estudio.

A continuación, se muestran 3 ejemplos de las construcciones dinámicas construidas, cada una según su propia ficha y explicada en base a su contenido.

5.1. Ejemplo 1: Puzle triángulo rectángulo isósceles. Esta de la relación pitagórica posee el código (4.I.A), que significa que es la ficha número 4 en orden ascendente de acuerdo al nivel académico al que pertenece, además que es una demostración de tipo geométrica (disección de áreas) y por último también se sabe que el uso de la construcción es más indicado para primer curso debido a la facilidad que presenta. Además de presentarlo como una construcción dinámica (Figura 1), este puzle también se puede realizar en madera u otros materiales y ser presentado a los estudiantes como un material concreto- manipulativo de geometría, tal como se plantea en (Barrantes, 1998) y en el que se elige trocear en piezas de puzles las figuras geométricas construidas sobre los cuadrados de los catetos de un triángulo rectángulo, para después recubrir con estas piezas la figura construida sobre la hipotenusa. 
Ficha Puzle 4: Triángulo rectángulo isósceles Código: 4. I.A

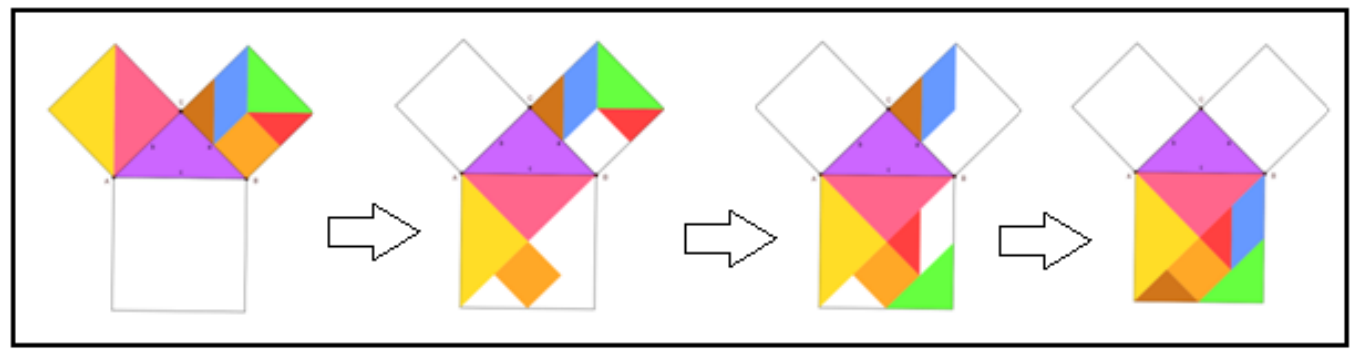

Figura 1. Momentos de la demostración para triángulos rectángulos isósceles.

Procedimiento: Podemos observar en la Figura 2, que las piezas del cuadrado de la hipotenusa corresponden a un tangram cuadrado. Luego basta con construir las piezas del geoplano cuadrado tomando como lado del cuadrado la hipotenusa. El triángulo rectángulo inicial, que aparece en morado, es el tamaño que se utiliza para generar los dos triángulos mayores del tangram. Así pues:

1. Se construye el triángulo rectángulo $A B C$ (morado).

2. Se construyen un tangram cuadrado de siete piezas de forma que el lado del cuadrado coincida con la hipotenusa.

3. Podemos resolver a partir del cuadrado de la hipotenusa o a partir de los cuadrados de los catetos.

4. En el segundo caso (momentos de la Figura 1), se trasladan los siete polígonos construidos para completar el cuadrado que está sobre el lado c del triángulo $A B C$.

Explicación: Esta demostración es válida solo en el caso de que el triángulo rectángulo inicial es isósceles por la construcción del tangram.

Demostración: Se puede hacer una demostración formal, hablando matemáticamente, pero en este caso hemos decidido hacer la demostración a partir de las propiedades del tangram.

Si tomamos como unidad de medida el triángulo pequeño rojo, se puede comprobar (por ejemplo superponiendo las piezas) que el cuadrado, el paralelogramo y el triángulo mediano miden 2 unidades y los triángulos

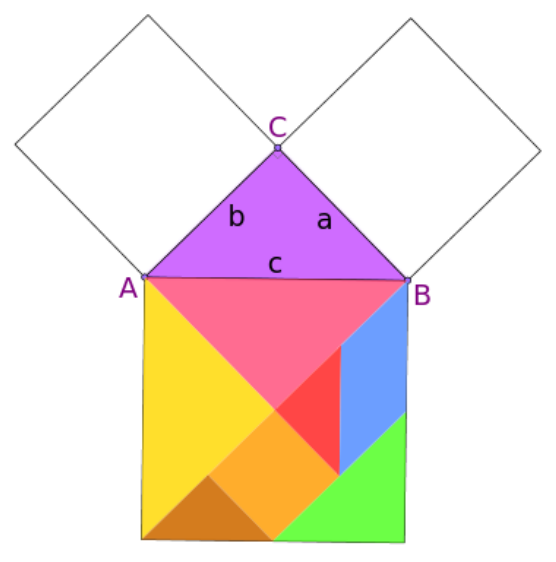

Figura 2. Puzle triángulo rectángulo isósceles. 
grandes 4 unidades. Luego el área del cuadrado sobre la hipotenusa sería 16 unidades. Tomando las medidas de los cuadrados sobre los catetos obtenemos que miden cada uno 8 unidades, lo que demuestra el teorema.

Este ejemplo, para su uso en el aula, tiene la ventaja que la demostración puede ser manipulada por el alumno mediante la sencilla construcción de un tangram cuadrado. De esta forma, la demostración mediante GeoGebra le va a ser mucho más fácil de comprender y asimilar porque ya la ha vivenciado manipulativamente. Esto es así, asumiendo que los estudiantes conocen lo que es un tangram.

5.2. Ejemplo 2: Demostración de Euclides. Esta demostración de la relación pitagórica posee el código (7.I.B), es una demostración de tipo geométrica (comparación de áreas equivalentes) indicada para el segundo curso. Es conocida como la demostración de Euclides y es clasificada en (Loomis, 1968) como la demostración número 33. Euclides fue el primer matemático en demostrar geométricamente el Teorema de Pitágoras. Dicha demostración se encuentra en su libro Los Elementos numerada como I.47. El alumno puede investigar también sobre dicho texto y saber que comienza con la definición de "punto" y termina con el inverso del Teorema de Pitágoras cuyo enunciado dice: Si la suma de los cuadrados de dos lados de un triángulo es igual al cuadrado del tercer lado, se trata de un triángulo recto. La demostración basada en equivalencia de triángulos, también, puede ser consultada en (Thomas, 1985).

\section{Procedimiento:}

1. Se construye el triángulo rectángulo $A B C$ (Figura 3).

2. Se construyen cuadrados sobre todos sus lados.

3. Se construye una recta perpendicular a la hipotenusa por el punto $C$, la intersección de esta recta con el segmento $A B$ se llama $P$ y con $G F$ se llama E.

4. Se dividen por la diagonal los cuadrados construidos sobre los catetos en dos triángulos semejantes a los construidos sobre el cuadrado de la hipotenusa (imagen C de la Figura 3).

5. Mediante estos triángulos semejantes, se traslada el cuadrado construido sobre el cateto mayor al rectángulo BPEG (Figura 4).

6. Igualmente se traslada el cuadrado construido sobre el cateto menor al rectángulo $P A F E$.

Explicación: El segmento $C P E$ divide el cuadrado construido sobre la hipotenusa del triángulo $A B C$ en dos rectángulos que tienen áreas iguales a las de los cuadrados construidos sobre los catetos del triángulo inicial. Es decir, el área del rectángulo $B P E G$ es igual al área del cuadrado construido sobre el cateto mayor y el área del rectángulo $P A F E$ es igual al área del cuadrado construido sobre el 
cateto menor del triángulo $A B C$. Es decir, el cuadrado de la hipotenusa es igual a la suma de los cuadrados construidos sobre los catetos.

\section{Ficha demostración de Euclides (Loomis 33) Código: 7.I.B}

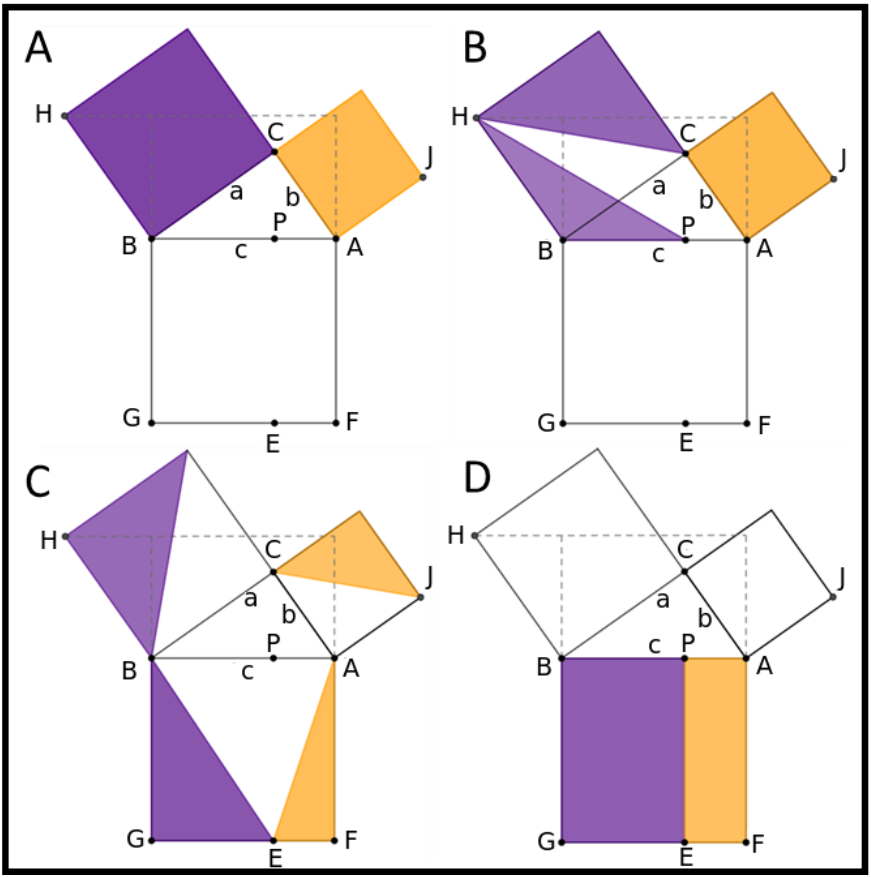

Figura 3. Momentos de la demostración de Euclides.

Demostración: Sean $a$ y $b$ los catetos del triángulo rectángulo $A B C$ y $c$ su hipotenusa, además, $A_{1}$ es la suma del área de los cuadrados construidos sobre los catetos y $A_{2}$ el área del cuadrado construido sobre la hipotenusa, esto es: $A_{1}=a^{2}+b^{2}$ y $A_{2}=c^{2}$.

Dividimos por la diagonal el cuadrado del cateto mayor obteniendo dos triángulos uno de los cuales es $\triangle B H C$. El triángulo $\triangle B H C$ tiene el mismo área que el triángulo porque la altura sobre la base $B H$ es la misma.Se puede comprobar que en los dos casos tienen la misma medida que el segmento $B C$ por construcción del triángulo rectángulo.

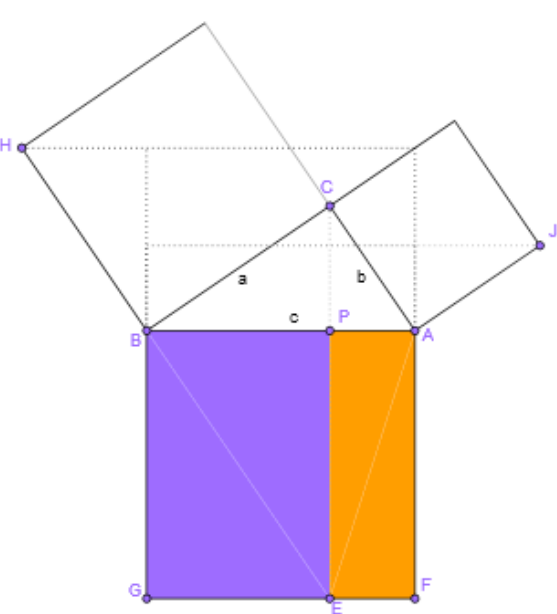

Figura 4. Demostración Euclides.

Se tiene que $\angle H B A=\angle C B G$ ya que cada uno de ellos es $\angle C B A+90^{\circ}$; esto implica que $\triangle H B A$ y $\triangle C B G$ son congruentes y su área es la misma área. También, el área de $\triangle C B G$ es igual al área de $\triangle B G P$ porque la altura sobre la base $B G$ es la misma. Se puede comprobar que en ambos casos es la medida del segmento $B P$. 
Luego el área de $\triangle H B C$ es igual al área de $\triangle B G P$. Haciendo lo mismo con el otro triángulo formado en el cateto mayor llegamos a que el rectángulo $B P E G$ es igual al cuadrado sobre el cateto mayor. Si dividimos ahora el cuadrado del cateto menor de igual forma, se llega a que el rectángulo $P A F E$ es igual al cuadrado sobre el cateto menor. Como $A_{1}=A_{2}$, se tiene que $a^{2}+b^{2}=c^{2}$.

El potencial de esta demostración, para su uso en el aula, está precisamente en su posibilidad de estudiar conjuntamente la historia de los matemáticos griegos, sus biografías y sus descubrimientos más notables para hacer comprender a los alumnos como se ha ido desarrollando la Geometría a lo largo del tiempo.

5.3. Ejemplo 3: Demostración de Bashkara. Tiene de código (19.II.C) y es del tipo algebraica para cuarto curso. Bhaskara es también conocido como Bhaskara II o como Bhaskaracharya, que significa "Bhaskara el maestro", nació en 1114, es probablemente el matemático indio de la antigüedad mejor conocido. Estaba dedicado a las matemáticas y a la astronomía y aportó una demostración sencilla del Teorema de Pitágoras en su trabajo Bijaganita (cálculo de raíces).

Ficha Bashkara (Loomis 36) Código:19. II.C

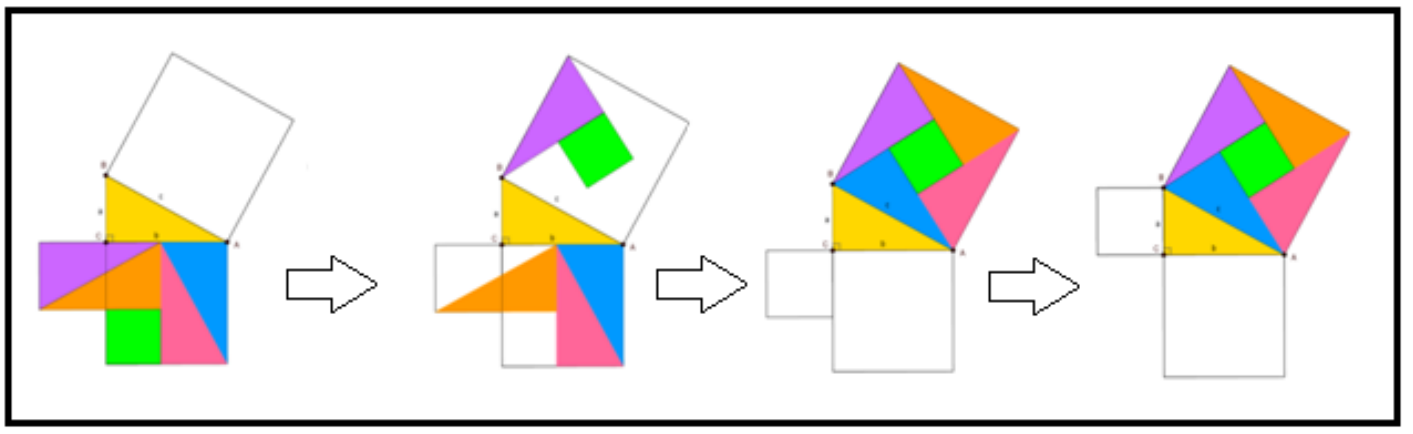

Figura 5. Momentos de la demostración de Bashkara (Loomis 36).

Procedimiento: El cuadrado sobre la hipotenusa se divide, como indica la Figura 5 (última imagen), en cuatro triángulos congruentes o equivalentes en área al dado y un cuadrado de lado igual a la diferencia de los catetos.

Explicación: Las piezas son reordenadas fácilmente para formar una figura que resulta ser la yuxtaposición de los cuadrados sobre los catetos. Esta demostración, también, puede ser utilizada en los primeros cursos de la Enseñanza Secundaria como una demostración geométrica del tipo partición de los cuadrados.

Demostración: Sean $a$ y $b$ los catetos del triángulo rectángulo $A B C$ (Figura 6) y $c$ su hipotenusa, además, $A_{1}$ es el área de los 4 triángulos de lados $a$ y $b$ más la 
del cuadrado (cuyo lado mide $b-a$ ) construidos inicialmente; y $A_{2}$ el área del cuadrado construido sobre la hipotenusa. Al comparar sus áreas se obtiene:

$$
\begin{aligned}
& A_{1}=4 \frac{a b}{2}+(b-a)^{2} \\
& A_{2}=c^{2}
\end{aligned}
$$

Como $A_{1}=A_{2}$, se obtiene que

$$
4 \frac{a b}{2}+(b-a)^{2}=c^{2}
$$

es decir $c^{2}=a^{2}+b^{2}$.
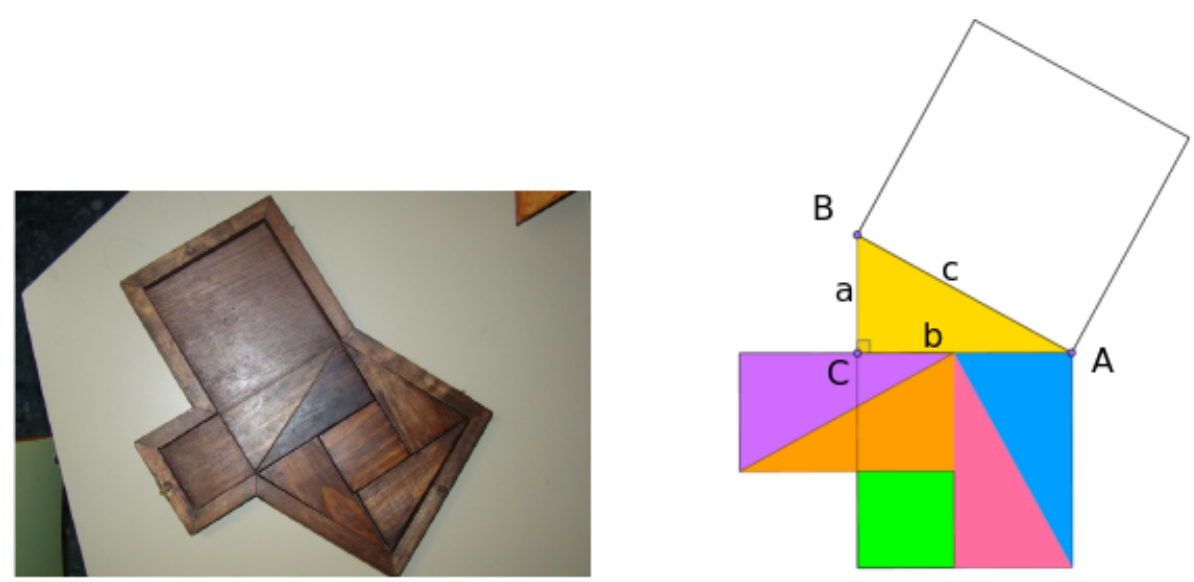

Figura 6. Demostración de Bashkara.

Esta demostración también puede ser manipulable (ver puzle Figura 6), como el primer ejemplo, por lo que la demostración con GeoGebra ya cuenta con la experiencia manipulativa del alumno. Desde el punto de vista histórico, este autor admite muchas curiosidades y anécdotas que completan el estudio teórico del tema. Así es recomendable su famoso libro Lilavati en el que además de su vida y obra, ofrece una gran variedad de problemas en verso, dedicados a su hija, y que comprenden varios temas de matemática básica y media que abarca aritmética, álgebra, combinatoria, geometría y trigonometría (Bhaskara, 2015), versión en español.

\section{$\S 6$. Conclusiones}

Hemos presentado un conjunto de construcciones dinámicas sobre las demostraciones geométricas y algebraicas del Teorema de Pitágoras, construidas con GeoGebra de manera selectiva, en base a una revisión actualizada y habiendo pasado por un proceso de clasificación y diseño, en el que se incluyeron elementos didácticos (procedimiento y explicación) para favorecer su uso en el proceso enseñanza y aprendizaje del teorema. 
Estas construcciones, consideramos, son un recurso importante para que el profesor trabaje la propiedad pitagórica de una manera activa y dinámica pudiendo además seleccionar, entra la gran variedad de demostraciones que ofrecemos, las que considere más acordes para el aprendizaje de sus alumnos.

Entendemos que el uso de software de geometría dinámica se ha convertido en un recurso que, combinado con el correcto uso del profesor, puede favorecer el aprendizaje en los alumnos debido al dinamismo en las construcciones, lo que permite que haya una interacción entre el conocimiento, el alumno y el profesor a través de las construcciones geométricas (Barrantes y Barrantes, 2017).

El estudio propone al alumnado diferentes demostraciones clásicas de la proposición pitagórica a la vez que se revaloriza la importancia de la demostración pitagórica, haciéndoles observar la cantidad de personas famosas, matemáticos o no, que se han preocupado por ella.

Las actividades dinámicas permiten explorar visualizaciones de la demostración pitagórica favoreciendo la construcción de conocimientos, a través de la manipulación directa del software de geometría dinámica; acciones que serían más trabajosas utilizando lápiz y papel. Los alumnos experimentan dificultades y logros de actividades que, aunque parecen actuales, han sido planteados y resueltos hace muchos siglos.

En futuros trabajos abordaremos las aplicaciones del Teorema de Pitágoras con el diseño de construcciones dinámicas que tengan que ver con problemas, juegos y curiosidades del mismo. Entre algunos de estos problemas, se pueden mencionar los cuadrados mágicos pitagóricos, las ternas pitagóricas, el problema del inverso del teorema, problemas de la historia de las Matemáticas, la generalización al espacio, el análogo del teorema en la geometría sólida y la relación entre el teorema y la sucesión de Fibonacci (Barrantes, 1998).

Evidenciamos de esta manera la importancia y posibilidades que tiene el Teorema de Pitágoras dando lugar a un sin número de demostraciones y aplicaciones que lo convierten en un auténtico problema abierto de la enseñanza y aprendizaje de la geometría.

La realización de estas actividades deben producir un cambio en las concepciones de los profesores y alumnos hacia una nueva mirada de la Geometría, distinta de la tradicional de libro y pizarra, en la que el alumno es el eje del aprendizaje de una materia básica en su vida diaria y laboral (Barrantes y Blanco, 2006).

\section{Bibliografía}

Alibert, D., y Thomas, M. (1991). Advanced mathematical thinking. Dordrecht, Países Bajos: Kluwer Academic Publishers. 
Alsina, C. (1997). ¿Por qué geometría? Propuestas didácticas para la ESO. Madrid: Síntesis.

Alsina, C. (2008). Geometría y realidad. Sigma: Revista de matemáticas.(33), 165-179.

Alsina, C., Fortuny, J., y Burgués, C. (1988). Materiales para construir la geometría. Madrid, España: Síntesis.

Barrantes, M. (1990). Pitágoras en el país de los puzles. Campo Abierto. Revista de Educación, 7(1), 221-230.

Barrantes, M. (1998). La geometría y la formación del profesorado en primaria y secundaria. Badajoz, España: Manuales UEX.

Barrantes, M., y Balletbo, I. (2012). La enseñanza y aprendizaje de la gometría en revistas científicas españolas de mayor impacto de la última década. San Juan Bautista, Paraguay: Litocolor.

Barrantes, M., y Barrantes, M. (2017). Geometría en la Educación Primaria. Badajoz, España: Indugraphic Digital.

Barrantes, M., y Blanco, L. (2006). A study of prospective Primary teacher's conceptions of teaching a learning geometry. Journal of Mathematics Teacher Education, 5(9), 411-436.

Bell, A. (1976). A study of pupil's proof-explanation in mathematical situation. Educational Studies in Mathematics, 1(7), 23-40.

Bergua, J. (1958). Pitágoras. Madrid, España: Ibéricas.

Bhaskara. (2015). Lilivati. Matemáticas en verso del siglo XII. Madrid, España: Real Sociedad Matemática Española S.M.

Cabrera, C., y Campistrous, L. (2007). Geometría dinámica en la escuela, ¿Mito o realidad? Uno: Revista de Didáctica de las Matemáticas, 13(45), 61-79.

Caniff, P. (1997). Pitágoras. Madrid, España: M.E. Editores.

De Villiers, M. (1993). El papel y la función de la demostración en matemática. Epsilon, 26, 15-29.

Duval, R. (1998). Perspectives on the Teaching of Geometry for the 21st Century. Dordrecht, Países Bajos: Kluwer Academic Publishers.

Fiallo, J. (2010). Estudio del proceso de demostración en el aprendizaje de las razones trigonométricas en un ambiente de geometría dinámica (Tesis Doctoral no publicada). Universitat de València.

González, P. (2001). Pitágoras. El filósofo del número. Madrid: Nivola.

González, P. (2004). La historia de las matemáticas como recurso didáctico e instrumento para enriquecer culturalmente su enseñanza. Suma, 45, 17-28.

González, P. (2008). El teorema de Pitágoras: Una historia geométrica de 4000 años . Sigma, 32, 103-130.

Groman, M. (1996). Integrating Geometer's Sketchpad into geometry couser for secondary education mathematics major.

Gutiérrez, A., y Jaime, A. (2012). Reflexiones sobre la enseñanza de la geometría en primaria y secundaria. Tecné, Episteme y Didaxis: TED., 32, 55-70. 
Hoyles, C. (1997). The curricular shaping of students' approaches. For the learning of Mathematics, 1(17), 7-16.

Jones, K. (2000). Providing oa foundation for deductive reasoning: students' interpretation when using dynamic geometry software and their evolving mathematical explanations. Educational Studies in Mathematics, 44, 55-85.

Lampert, M. (1990). When the problem is not the question and the solution is not the answer: mathematical knowing and teaching. American Educational Research Journal, 27(1), 29-63.

Loomis, E. (1968). The Pythagorean Proposition. Washington, D.C.: National Council of Teachers of Mathematics.

Mariotti, M. (2000). Proofs produced by secondary school students learning geometry in a dynamic computer environmet. Educational Studies in Mathematics, $44,87-125$.

Marrades, R., y Gutiérrez, A. (2000). Proofs produced by secondary school students learning geometry in a dunamic computer environmmet. Educational Studies in Matematics(44), 87-125.

Nelsen, R. (1993). Proof without word: Exercises in visual thinking. Washington, D.C., EEUU: The Mathematical Association of America.

Sada, M. (2010). Algunas de las posibilidades didácticas de GeoGebra en las aulas. II Jornadas de Integración de las TIC en la Enseñanza.

Schuré, E. (1995). Los grandes iniciados. Vol. II. Buenos Aires, Argentina: REI.

Strathern, P. (1999). Pitágoras y su teorema. Madrid, España: Siglo XXI de España Editores.

Thomas, I. (1985). Matemáticos griegos.Enciclopedia Sigma I. Barcelona, España: Grijalbo.

Vasquez, M. (2012). Una ampliación al teorema de Pitágoras. Revista de Educación Matemática, 27(3), 3-22.

María Consuelo Barrantes Masot

Universidad de Valencia. España.

(两) conbarmas@gmail.com

Victor ZAMORA RoDRíGUeZ

Universidad de Extremadura. España.

(凹) victor@unex.es

MANuel Barrantes López

Universidad de Extremadura. España.

(凶) barrante@unex.es

Recibido: 23 de marzo de 2020.

Aceptado: 11 de noviembre de 2020.

Publicado en línea: 5 de abril de 2021. 